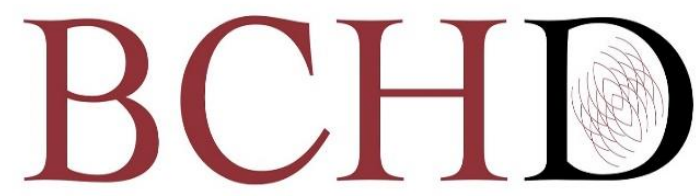

Bioactive Compounds in Health and Disease

\title{
The effect of low level-laser irradiation on antioxidant enzymes and mineral levels in serum of patients with type 2 diabetes mellitus
}

\author{
Danik Martirosyan ${ }^{1}$, Mohammad Reza Ashoori ${ }^{2}$, Hossein Mirmiranpour ${ }^{3 *}$
}

${ }^{1}$ Functional Food Center, Functional Food Institute, Dallas, TX, USA; ${ }^{2}$ Department of Laboratory Sciences, School of Health and Allied Medical Sciences, Zanjan University of Medical Sciences, Zanjan, Iran; ${ }^{3}$ Endocrinology and Metabolism Research Center (EMRC), Vali-Asr Hospital, School of Medicine, Tehran University of Medical Sciences, Tehran, Iran

*Corresponding Author: Hossein Mirmiranpour, MD, PhD, Endocrinology and Metabolism Research Center (EMRC), Vali-Asr Hospital, School of Medicine, Tehran University of Medical Science, 1th floor, Keshavarz Boulevard, Tehran, Iran.

Submission Date: April 1st, 2020; Acceptance Date: May 22nd, 2020; Publication Date: May 27th, 2020

Please cite this article as: Martirosyan D., Ashoori M. R., Mirmiranpour H. The effect of low level-laser irradiation on antioxidant enzymes and mineral levels in serum of patients with type 2 diabetes mellitus. Bioactive Compounds in Health and Disease.

2020; 3(5): 82-96. DOI: https:/doi.org/10.31989/bchd.v3i5.705

\section{ABSTRACT}

Background: The control and management of type 2 diabetes mellitus is the most important way to prevent health consequences of the disease. Oxidative stress derived from diabetes mellitus is an important cause of these symptoms in uncontrolled diabetic patients. The consumption of functional foods containing antioxidants and trace minerals can help prevent these consequences and control diabetes mellitus.

Objective: In this study, we examined whether low-level laser therapy could have an effect on levels of antioxidant enzymes and minerals in the serum of patients with type 2 diabetes mellitus.

Methods: Thirty individuals with type 2 diabetes and thirty healthy individuals, as controls, were selected as participants for this study. The levels of antioxidant enzymes glutathione peroxidase, catalase, superoxide dismutase; biochemical parameters, such as glucose and hydrogen peroxide; minerals iron, zinc, magnesium, copper; and selenium binding protein 1 (as an indicator of selenium) were studied before and after low level-laser therapy.

Results: The levels of antioxidant enzymes and some minerals significantly increased in control and diabetic patients after low level-laser therapy. The levels of glucose, hydrogen peroxide, and selenium binding protein 1 , however, were unchanged after low-level laser therapy.

Conclusions: Low level-laser therapy may be an important tool for reducing oxidative stress caused by type 2 diabetes mellitus due to its ability to increase levels of antioxidant enzymes and mineral content. 
Keywords: Laser Irradiation; Antioxidant; Functional Foods/Bioactive Compounds; Diabetes Mellitus.

CFFC 2020. This is an Open Access article distributed under the terms of the Creative Commons Attribution 4.0 License (http://creativecommons.org/licenses/by/4.0)

\section{INTRODUCTION}

Diabetes mellitus (DM) is one of the most prevalent chronic metabolic diseases worldwide and is characterized by elevated levels of glucose in blood (hyperglycemia). According to the World Health Organization (WHO), 422 million people lived with diabetes in 2014 [1, 2]. Moreover, diabetes caused 1.5 million deaths in 2012 [3]. There are two main types of the disease, type 1 diabetes mellitus (T1DM) and type 2 diabetes mellitus (T2DM). T2DM results from insulin resistance or an inability to produce enough insulin. This type of diabetes is associated with poor nutrition, an unhealthy lifestyle, and low physical activity. Hyperglycemia induced by T2DM causes a rise in reactive oxygen species (ROS) production which can lead to oxidative stress and impairs the antioxidant defense system [4,5]. Oxidative stress and inflammation caused by diabetes may produce symptoms and certain health consequences of DM [6] such as skin wounds, peripheral neuropathy, nephropathy, and retinopathy [7]. The role of oxidative stress, inflammatory responses, and cell signaling have been considered in causing these consequences [8].

Functional foods have been found to be essential for the management and prevention of certain diseases such as diabetes and its sequelae [9]. Functional foods can also be sources of many micronutrients such as vitamins and minerals, which have antioxidant and anti-inflammatory properties [10]. Minerals found in functional foods are especially important for the antioxidant defense system. Antioxidants enzymes such as glutathione peroxidase (GPX), superoxide dismutase (SOD), and catalase (CAT) can be extracted from some functional foods and bioactive compounds [11-13]. These enzymes found in antioxidants are the first line of defense in the human body against oxidative stress and depend on trace minerals such as Zinc (Zn), Selenium (Se), Iron (Fe), Copper ( $\mathrm{Cu})$, and Magnesium (Mg) as cofactors [14]. These enzymes play a vital role in protecting the body's biological systems against attack by free radicals [14]. It is interesting to note that that there is mounting attention in investigating these antioxidants' preventative and therapeutic abilities against disease.

Photo biomodulation, or low level-laser therapy (LLLT), has been used for more than fifty years [15]. This kind of laser, instead of producing any heat, acts through photo-chemical and photo-physical reactions [16]. Currently, LLLT appears to be an increasingly popular therapeutic option among physicians for many problems such as skin wounds and neuropathic pains [17-21]. For example, low-energy laser radiation has exerted direct and considerable influence on tissue repair processes without causing heating effects [22]. In clinical settings, LLLT has been used for accelerating healing, regeneration, and reducing pain and inflammation $[23,24]$. LLLT has also shown to be effective in healing skin wounds by stimulating fibroblasts and cell proliferation as well as collagen production [25]. Researchers have suggested that low level-laser therapy (LLLT) may influence oxidative stress parameters as well as alter the activity of antioxidant enzymes and output of ROS [26]. Thus, LLLT may have importance in the control of DM in the patients with T2DM.

This study reviews the effect of LLLT on the activity of several antioxidant enzymes and their trace element cofactors. The evaluation of LLLT's effect on these minerals as a group of bioactive compounds was another goal of this investigation. A correlation between LLLT use and changes in these minerals' quantity and alterations to above-mentioned enzymes' function may facilitate new approaches to antioxidant activation in humans. Therefore, the aim of this study is to assess the effect of LLLT on the activity of antioxidant enzymes, serum quantity of antioxidant minerals, and related correlations between these enzymes and minerals in T2DM.

\section{METHODS}

Diagnostic kits for the antioxidant enzymes CAT and GPx were purchased from Biocore (Biocore 
Diagnostik Ulm GmbH, Ulm, Deutschland). The SOD assay kit was purchased from Biovision (BioVision Incorporated, USA). The hydrogen peroxide $\left(\mathrm{H}_{2} \mathrm{O}_{2}\right)$ assay kit was purchased from ZellBio (ZellBio $\mathrm{GmbH}$, Ulm, Germany). Human glucose (with an intra- and inter-assay $\mathrm{CV}<8 \%$ and $<10 \%$, respectively) enzymelinked immunosorbent assay (ELISA) kits were purchased from MyBioSource Inc. (San Diego, CA 92195-3308, USA). Zn, Cu, Fe, Mg and Se binding protein 1 assay kits were purchased from MyBioSource Inc. (San Diego, CA 92195-3308, USA). Parameters were evaluated according to kit instructions. Microplate reader (Mindray, model MR96A, Germany) and microplate spectrophotometer (model Fluostar, bmglabtech, Germany) were used for this assay. Green diode pumped solid state (DPSS) Laser Pointer (model RLP-532, 1040 Vienna, Austria) was used for LLLT.

Participants: In this research, thirty patients with T2DM and thirty healthy individuals, as a control group, were randomly selected among the persons referred to the Endocrinology and Metabolism Research Center (EMRC), Vali-Asr Hospital, Tehran University of Medical Sciences, Tehran, Iran. Written consent was obtained from healthy subjects and persons with T2DM before sampling and starting the study. People with other chronic illnesses, people undergoing surgery, and those with T1DM and infectious illness were excluded. Diagnostic criteria for T2DM was accomplished according to WHO standards for DM.

General Characteristics and Sampling: In this study, the subjects were divided into two groups: those with T2DM and healthy controls. General characteristics of participants such as sex, age, height, weight and body mass index (BMI) were recorded. Blood samples were obtained from all participants after 12 hours of overnight fasting. Blood samples were centrifuged, and their serum was separated. The obtained serum was used to evaluate the parameters described above. After 24 hours, samples were irradiated by low level green LASER diode and then examined for a second time.

Laser Irradiation: A diode laser pointer was used for irradiation. The laser was a low level green laser diode with a wavelength of $532 \mathrm{~nm}$ at $100 \mathrm{mw}$ in a continuous wave mode with divergence $<1.5 \mathrm{mRad}$, beam mode (TEMoo), beam diameter at aperture 1.5, crystal type Nd:VYO4:KTP, and power source $1 \times$ 3V CR2 alkaline batteries. The power density was $509.55 \mathrm{~mW} / \mathrm{cm} 2$ at a distance of $6.5 \mathrm{~cm}$ from the laser device from serum inside the tube, and the diameter of the laser spot was set to $0.5 \mathrm{~cm}$. Irradiation was applied for 8 seconds.

Statistical Analysis: Statistical analysis was accomplished utilizing SPSS (version 23, IBM, USA) software for Windows. All results were expressed as mean \pm standard deviation. Paired sample t-test and independent sample t-test were used to compare the mean of the obtained data. In all analyses, a p-value of $<0.05$ qualified the presence of a statistically substantial difference.

\section{RESULTS}

General Characteristics: The age of participants in this study was between 55 and 75 years old. Among healthy controls, $60 \%$ were female and $40 \%$ were male. $37 \%$ of people with T2DM were female and $63 \%$ of people with T2DM were male. General characteristics between the control group and diabetic group are shown in Table 1 . In the diabetic group, height, weight, and body mass index (BMI) were not significant in comparison with the healthy control group.

Table 1. Participant's General Characteristics

\begin{tabular}{cccc}
\hline Characteristic & $\begin{array}{c}\text { Control } \\
\text { Group } \\
\mathbf{n}=\mathbf{3 0}\end{array}$ & $\begin{array}{c}\text { Diabetic } \\
\text { Group } \\
\mathbf{n}=\mathbf{3 0}\end{array}$ & P value \\
\hline Height $(\mathrm{Cm})$ & $165.3 \pm 5.6$ & $165.8 \pm 6.6$ & 0.72 \\
Weight $(\mathrm{Kg})$ & $78.2 \pm 6.2$ & $79.9 \pm 8.0$ & 0.35 \\
BMI $\left(\mathrm{Kg} / \mathrm{m}^{2}\right)$ & $28.6 \pm 2.1$ & $29.0 \pm 1.5$ & 0.47 \\
\hline
\end{tabular}

Data are given as mean \pm SD. P value $<0.05$ is significant. BMI, body mass index

Biochemical Parameters: The levels of antioxidant enzymes CAT, SOD, and GPx were quantified in the collected samples. The levels of aforementioned minerals associated with these enzymes as well as glucose and $\mathrm{H}_{2} \mathrm{O}_{2}$ were also measured. The 
concentrations of these parameters were measured before and after LLLT (Table 2) and compared in the control and diabetic groups. The results of this study are shown in Table 3.

As seen in Table 2, there was a significant difference in the concentrations of catalase, GPx, and SOD in the control and diabetic groups before and after LLLT ( $P$ value < 0.001). Comparison of glucose, $\mathrm{H}_{2} \mathrm{O}_{2}$, and Sebp1 concentrations did not show any significant difference in the control and diabetic groups before and after LLLT ( $P$ value $>0.05$ ). There was also no significant difference in the comparison of concentrations of minerals ( $\mathrm{Fe}, \mathrm{Zn}, \mathrm{Mg}$ and $\mathrm{Cu}$ ) in the control group before and after LLLT ( $P$ value > $0.05)$. However, there was a significant difference in the concentration of these minerals in the diabetic group before and after LLLT ( $P$ value was 0.01, 0.005, 0.001 , and 0.001, respectively for $\mathrm{Fe}, \mathrm{Cu}, \mathrm{Mg}$ and $\mathrm{Zn}$ ). The comparison of mean concentration between all biochemical parameters in the control and diabetic groups after LLLT showed a significant difference ( $P$ value <0.001).

The relationship between the intake of these bioactive compounds, diabetes, and LLLT as well as changes in the concentration of antioxidant enzymes and minerals is summarized in Figure 1.

\section{Diabetes mellitus}
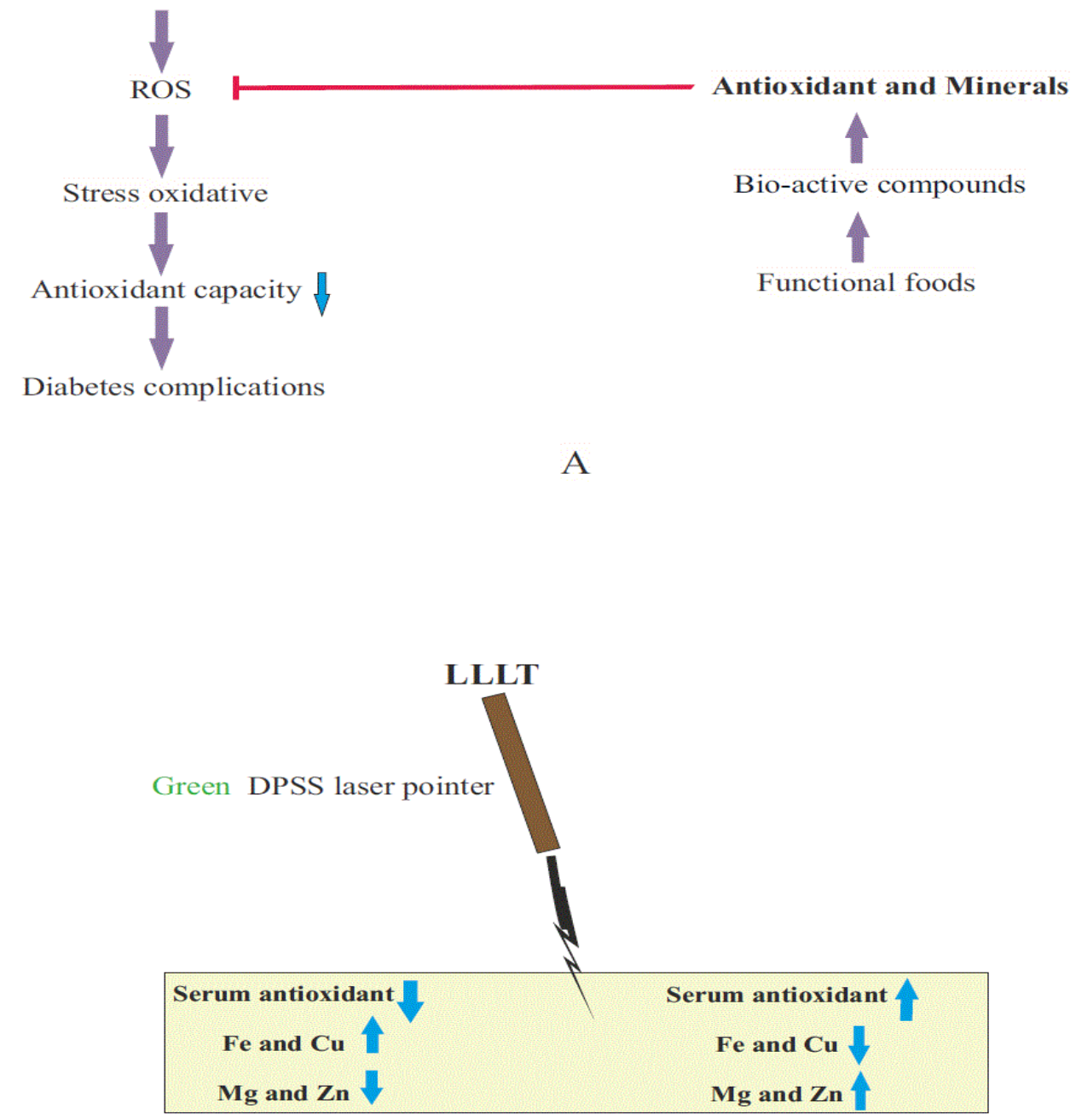

B

Figure 1. The relationship between diabetes, antioxidants, and LLLT. (A) The relationship between functional foods intake, antioxidants, and reduction of oxidative stress formation by inhibition of reactive oxygen species. (B) The effect of LLLT on antioxidant enzymes and some minerals in the serum of diabetic patients before and after LLLT. 


\section{DISCUSSION}

In this study, we aimed to investigate the effects of LLLT on the quantity of several biochemical parameters in patients with T2DM. The therapeutic effect of LLLT on diabetic patients has been evaluated in many studies. In one study by Denadai et al., it was reported that LLLT (660 $\mathrm{nm}$ ) was effective in reducing levels of oxidative stress in diabetic rats [27]. In one of our previous studies, the effect of LLLT on the activity of CAT was investigated. In that study, we found that green $(530 \mathrm{~nm})$ and blue $(450 \mathrm{~nm})$ laser light increased CAT activity in blood samples more than red laser light [28].

In terms of increased CAT activity, this study agrees with our previous study. Lim et al. also concluded that LLLT $(670 \mathrm{~nm})$ increased CAT activity and expression. In their experiments, LLLT had no effect on GPx and SOD activity. [29] In this study, no reduction in $\mathrm{H}_{2} \mathrm{O}_{2}$ by LLLT was observed in the control and diabetic groups. However, GPx and SOD concentrations increased significantly in both the control and diabetic groups. By reducing oxidative stress through increasing the activity of antioxidant enzymes, such as CAT and SOD, health consequences of diabetes may be reduced. LLLT may be an effective method to increase the activity of antioxidant enzymes, according to previous studies and the results of this study.

In our study, LLLT had no effect on lowering blood glucose, which also agrees with the study by Lim et al. However, Longo et al. reported that LLLT is effective in lowering blood glucose for patients with T1DM [30].

According to the new definition of functional foods by the Functional Foods Center (FFC) [31], any functional food product contains one or more biologically active compounds such as minerals, vitamins, and antioxidant enzymes. The therapeutic effects of some dietary supplements containing such biologically active compounds, which have anti-

Table 2. Comparison between the concentrations of biochemical parameters, before and after LLLT, in two groups

\begin{tabular}{|c|c|c|c|c|c|c|}
\hline \multirow[b]{2}{*}{ Parameters } & \multicolumn{3}{|c|}{$\begin{array}{c}\text { Control Group } \\
n=30\end{array}$} & \multicolumn{3}{|c|}{$\begin{array}{c}\text { Diabetic Group } \\
n=30\end{array}$} \\
\hline & $\begin{array}{c}\text { Before } \\
\text { LLLT }\end{array}$ & After LLLT & $P$ value & $\begin{array}{c}\text { Before } \\
\text { LLLT }\end{array}$ & $\begin{array}{l}\text { After } \\
\text { LLLT }\end{array}$ & $P$ value \\
\hline CAT (U/ml) & $2.7 \pm 0.07$ & $2.9 \pm 0.03$ & $<0.001$ & $1.5 \pm 0.2$ & $2.6 \pm 0.2$ & $<0.001$ \\
\hline GPx (U/ml) & $121.8 \pm 2.3$ & $122.7 \pm 2.4$ & $<0.001$ & $81.5 \pm 6.2$ & $83.8 \pm 6.3$ & $<0.001$ \\
\hline SOD (U/ml) & $7.6 \pm 0.3$ & $7.8 \pm 0.3$ & $<0.001$ & $4.0 \pm 0.3$ & $4.8 \pm 0.4$ & $<0.001$ \\
\hline Glucose $(\mu \mathrm{g} / \mathrm{ml})$ & $302.6 \pm 19.5$ & $301.8 \pm 19.0$ & 0.86 & $424 \pm 23.1$ & $420 \pm 20.9$ & 0.57 \\
\hline $\mathrm{H} 2 \mathrm{O} 2(\mu \mathrm{M} / \mathrm{ml})$ & $223.6 \pm 19.6$ & $221.8 \pm 19.6$ & 0.71 & $332.3 \pm 27.5$ & $323 \pm 23.0$ & 0.23 \\
\hline Sebp1 (pg/ml) & $4971.1 \pm 289.6$ & $4976.1 \pm 289.6$ & 0.95 & $3171.1 \pm 289.6$ & $3221.2 \pm 289.8$ & 0.54 \\
\hline $\mathrm{Fe}(\mu \mathrm{mol} / \mathrm{l})$ & $292.3 \pm 11.3$ & $291.3 \pm 11.2$ & 0.74 & $397.6 \pm 14.3$ & $387.3 \pm 14.4$ & 0.01 \\
\hline $\mathrm{Cu}(\mu \mathrm{mol} / \mathrm{I})$ & $295.3 \pm 12.3$ & $294.2 \pm 12.2$ & 0.66 & $399.4 \pm 13.9$ & $389.3 \pm 13.9$ & 0.005 \\
\hline Mg (mmol/l) & $7.0 \pm 0.8$ & $7.1 \pm 0.7$ & 0.47 & $3.9 \pm 0.8$ & $4.7 \pm 0.9$ & 0.001 \\
\hline $\mathrm{Zn}(\mu \mathrm{mol} / \mathrm{I})$ & $155.8 \pm 6.6$ & $156.9 \pm 6.5$ & 0.51 & $99.1 \pm 5.9$ & $105.2 \pm 5.9$ & 0.001 \\
\hline
\end{tabular}

Results are showed as mean \pm SD. P value $<0.05$ is significant. LLLT, low level-laser therapy; CAT, catalase; GPx, glutathione peroxidase; SOD, superoxide dismutase; Sebp1, selenium binding protein 1' Fe. Iron; Cu, Copper; Mg, Magnesium; Zn, Zinc. 
Table 3. Comparison between the concentrations of biochemical parameters after LLLT in two groups.

\begin{tabular}{|c|c|c|c|}
\hline Parameters & $\begin{array}{c}\text { Control Group } \\
n=30\end{array}$ & $\begin{array}{c}\text { Diabetic Group } \\
\qquad n=\mathbf{3 0}\end{array}$ & $P$ value \\
\hline CAT (U/ml) & $2.9 \pm 0.03$ & $2.6 \pm 0.2$ & $<0.001$ \\
\hline GPx (U/ml) & $122.7 \pm 2.4$ & $83.8 \pm 6.3$ & $<0.001$ \\
\hline SOD (U/ml) & $7.8 \pm 0.3$ & $4.8 \pm 0.4$ & $<0.001$ \\
\hline Glucose $(\mu \mathrm{g} / \mathrm{ml})$ & $301.8 \pm 19.0$ & $420 \pm 20.9$ & $<0.001$ \\
\hline $\mathrm{H} 2 \mathrm{O} 2(\mu \mathrm{M} / \mathrm{ml})$ & $221.8 \pm 19.6$ & $323 \pm 23.0$ & $<0.001$ \\
\hline Sebp1 (pg/ml) & $4976.1 \pm 289.6$ & $3221.2 \pm 289.8$ & $<0.001$ \\
\hline $\mathrm{Fe}(\mu \mathrm{mol} / \mathrm{l})$ & $291.3 \pm 11.2$ & $387.3 \pm 14.4$ & $<0.001$ \\
\hline $\mathrm{Cu}(\mu \mathrm{mol} / \mathrm{l})$ & $294.2 \pm 12.2$ & $389.3 \pm 13.9$ & $<0.001$ \\
\hline $\mathrm{Mg}(\mathrm{mmol} / \mathrm{l})$ & $7.1 \pm 0.7$ & $4.7 \pm 0.9$ & $<0.001$ \\
\hline $\mathrm{Zn}(\mu \mathrm{mol} / \mathrm{l})$ & $156.9 \pm 6.5$ & $105.2 \pm 5.9$ & $<0.001$ \\
\hline
\end{tabular}

Results are showed as mean \pm SD. P value $<0.05$ is significant. LLLT, low level-laser therapy; CAT, catalase GPx, glutathione peroxidase; SOD, superoxide dismutase; Sebp1, selenium binding protein 1; Fe, Iron; Cu, Copper; Mg, Magnesium; Zn, Zinc.

inflammatory and antioxidant properties have been discussed [32]. Thus, functional foods containing such compounds may be effective in managing diabetes and other chronic diseases [34]. Zn, which could be considered as a bioactive compound in functional food, is one of the essential trace elements in humans and $\mathrm{Zn}$ deficiency has been observed in diseases such as DM [35]. Zn acts as an insulin mimetic in the insulin signal transduction pathway and therefore has insulin-like effects in glucose homeostasis [36]. In one study, Abdel-Magied et al. investigated the effect of LLLT $(870 \mathrm{~nm})$ on certain minerals and enzymes in the liver and kidney of rats. They reported that LLLT increased the activity of antioxidant enzymes such as SOD and CAT as well as the abundance of minerals such as $\mathrm{Fe}, \mathrm{Cu}, \mathrm{Zn}, \mathrm{Mg}$, and Se [37]. Selenium can act as an anti-inflammatory and antioxidant factor, and acts as a cofactor in selenoproteins such as SOD [38]. We studied Sebp1 as an indicator of Se concentration. In our study LLLT significantly increased levels of $\mathrm{Zn}$ and $\mathrm{Mg}$ in diabetic patients, but decreased levels of $\mathrm{Cu}$ and Fe. The concentration of Sepb1 was not significantly different in diabetic and control subjects before and after LLLT.
However, the difference in levels of certain minerals and enzymes was significant between control and diabetic subjects after LLLT. In a study by Atalay et al. [38] where several trace elements were studied in the serum of women with T2DM, it was suggested that levels of minerals such as $\mathrm{Mg}, \mathrm{Fe}, \mathrm{Cu}$, and $\mathrm{Zn}$ were low in women with T2DM. These differences in mineral levels between diabetic and non-diabetic individuals may express the relationship between glucose metabolism and these minerals. Low Mg levels, for example, are associated with poor glycemic control [39]. According to the above results and the results of our study, the activity of antioxidant enzymes as well as trace elements should be considered in diabetic patients. LLLT and the consumption of functional foods containing antioxidant enzymes and trace minerals may play an important role in increasing the levels of these parameters.

\section{CONCLUSION}

Our study showed that LLLT use in patients with T2DM may be used to increase levels of antioxidant enzymes and minerals and alleviate diabetes- 
associated oxidative stress. Intake of bioactive compounds such as $\mathrm{Mg}, \mathrm{Zn}, \mathrm{Se}, \mathrm{Fe}$, and $\mathrm{Cu}$ through functional foods may also play an important role in controlling DM and preventing its health consequences.

\section{LIST OF ABBREVIATIONS}

LLLT, low level-laser therapy; DM, diabetes mellitus; T1DM, type 1 diabetes mellitus; T2DM, type 2 diabetes mellitus; WHO, world health organization; GPx, glutathione peroxidase; SOD, superoxide dismutase; CAT, catalase; ROS, reactive oxygen species; BMI, body mass index; Sebp1, selenium binding protein 1; Fe, Iron; Zn, Zinc; Mg, Magnesium; $\mathrm{Cu}$, Copper.

\section{ACKNOWLEDGMENTS}

The authors of this article sincerely acknowledge the staff at Endocrinology and Metabolism Research Center.

\section{COMPETING INTERESTS}

The authors declare that there are no conflicts of interest.

\section{AUTHOR'S CONTRIBUTIONS}

Danik Martirosyan participated in the study design. He also edited the article. Mohammad Reza Ashoori participated in the writing and analysis of the results. Hossein Mirmiranpour contributed to the original idea of the paper, doing the experimental work and, data collection. All authors read and approved the final version before its submission.

\section{HUMAN AND ANIMAL STUDIES}

This article contains human studies. All of which were consented and humane. No animal studies were conducted.

\section{REFERENCES}

1. Ashoori, M.R., M. Rahmati-Yamchi, A. Ostadrahimi, R. Pahlavan-Gharebaba, M. Mobasseri, S. Bakhtiyari, et al.: Apelin-13 serum levels in type 2 diabetic obese women: possible relations with microRNAs-107 and 375. Turk Biokim Derg, 2019. 44(5), 667-675.

2. Ghafarian-Alipour, F., S. Ziaee, M.R. Ashoori, M.S. Zakeri, M.A. Boroumand, N. Aghamohammadzadeh, et al.: Association between FTO gene polymorphisms and type 2 diabetes mellitus, serum levels of apelin and androgen hormones among Iranian obese women. Gene, 2018. 641, 361-366.

3. Organization, W.H., Global report on diabetes: executive summary. 2016, World Health Organization.

4. Sruthi, G., H.H. Pillai, N. Ullas, V. Jiju, and E. Abraham: Role of Antioxidants in the Management Diabetes Mellitus. International J Pharma Nanotech, 2017. 10, 15.

5. Morais, J.B.S., J.S. Severo, L.R. Dos Santos, S.R. de Sousa Melo, R. de Oliveira Santos, A.R.S. de Oliveira, et al.: Role of magnesium in oxidative stress in individuals with obesity. Biol Trace Elem Res, 2017. 176(1), 20-26.

6. Pickering, R.J., C.J. Rosado, A. Sharma, S. Buksh, M. Tate, and J.B. de Haan: Recent novel approaches to limit oxidative stress and inflammation in diabetic complications. Clin Trans Immunology, 2018. 7(4), e1016.

7. Forbes, J.M. and M.E. Cooper: Mechanisms of diabetic complications. Physiol Rev, 2013. 93(1), 137-188.

8. Giacco, F. and M. Brownlee: Oxidative stress and diabetic complications. Circ Res, 2010. 107(9), 10581070.

9. Li, Y., Y. Liu, J. Liang, T. Wang, M. Sun, and Z. Zhang: Gymnemic Acid Ameliorates Hyperglycemia through PI3K/AKT-and AMPK-Mediated Signaling Pathways in Type 2 Diabetes Mellitus Rats. J Agric Food Chem, 2019. 67(47), 13051-13060.

10. Serafini, M. and I.J.C.P.D. Peluso: Functional foods for health: the interrelated antioxidant and antiinflammatory role of fruits, vegetables, herbs, spices and cocoa in humans. 2016. 22(44), 6701-6715.

11. Xu, D.-P., Y. Li, X. Meng, T. Zhou, Y. Zhou, J. Zheng, et al.: Natural antioxidants in foods and medicinal plants: Extraction, assessment and resources. 2017. 18(1), 96.

12. Zemestani, M., M. Rafraf, and M. Asghari-Jafarabadi: Chamomile tea improves glycemic indices and antioxidants status in patients with type 2 diabetes mellitus. Nutrition, 2016. 32(1), 66-72.

13. Panahi, Y., M.S. Hosseini, N. Khalili, E. Naimi, M. Majeed, and A.J.C.n. Sahebkar: Antioxidant and antiinflammatory effects of curcuminoid-piperine combination in subjects with metabolic syndrome: a randomized controlled trial and an updated metaanalysis. 2015. 34(6), 1101-1108.

14. Ighodaro, $O$. and O. Akinloye: First line defence antioxidants-superoxide dismutase (SOD), catalase (CAT) and glutathione peroxidase (GPX): Their fundamental role in the entire antioxidant defence grid. Alexandria J Med, 2018. 54(4), 287-293.

15. Moskvin, S.V.: Low-level laser therapy in Russia: history, science and practice. J Lasers Med Sci, 2017. 8(2), 56.

16. Amjadi, A., H. Mirmiranpour, S.O. Sobhani, and N.M. Goudarzi: Intravenous laser wavelength radiation effect on LCAT, PON1, catalase, and FRAP in diabetic rats. Lasers in medical science, 2020. 35(1), 131-138.

17. Lopes-Martins, R.A.B., R.L. Marcos, E.C.P. Leal-Junior, and J.M. Bjordal, Low-Level laser therapy and world association for laser therapy dosage recommendations in musculoskeletal disorders and injuries. 2018, Mary 
Ann Liebert, Inc. 140 Huguenot Street, 3rd Floor New Rochelle, NY 10801 USA.

18. Ahmed, O.F., A.M. Elkharbotly, N. Taha, and A.B. Bekheet: Treatment of mild to moderate carpal tunnel syndrome in patients with diabetic neuropathy using low level laser therapy versus ultrasound controlled comparative study. BBA Clin, 2017. 8, 43-47.

19. de Andrade, A.L.M., P.S. Bossini, and N.A. Parizotto: Use of low level laser therapy to control neuropathic pain: a systematic review. Journal of Photochemistry and Photobiology B: Biology, 2016. 164, 36-42.

20. Zahra Al Timimi, M. and M.Z.M. Jafri: Photodynamic therapy and green laser blood therapy. Glob Jl Med Res, 2011. 11(5).

21. Abdel-Wahhab, K.G., E.M. Daoud, A. El Gendy, H.H. Mourad, F.A. Mannaa, and M.M. Saber: Efficiencies of low-level laser therapy (LLLT) and gabapentin in the management of peripheral neuropathy: diabetic neuropathy. App Biochem Biotechno, 2018. 186(1), 161-173.

22. Assis, L., C. Manis, K.R. Fernandes, D. Cabral, A. Magri, S. Veronez, et al.: Investigation of the comparative effects of red and infrared laser therapy on skeletal muscle repair in diabetic rats. Am J Phys Med Rehabil, 2016. 95(7), 525-534.

23. Avci, P., A. Gupta, M. Sadasivam, D. Vecchio, Z. Pam, N. Pam, et al. Low-level laser (light) therapy (LLLT) in skin: stimulating, healing, restoring. in Seminars in cutaneous medicine and surgery. 2013. NIH Public Access.

24. SO, S.: Intravenous Laser Wavelength Irradiation Effect on Interleukins: IL-1 $\alpha$, IL-1 $\beta$, IL6 in Diabetic Rats. LASER THERAPY, 2019. 28(4), p. 267-273.

25. Tatmatsu-Rocha, J.C., C. Ferraresi, M.R. Hamblin, F.D. Maia, N.R.F. do Nascimento, P. Driusso, et al.: Low-level laser therapy (904 $\mathrm{nm}$ ) can increase collagen and reduce oxidative and nitrosative stress in diabetic wounded mouse skin. J Photochem Photobiol B, 2016. 164, 96102.

26. Mathur, R., K. Sahu, S. Saraf, P. Patheja, F. Khan, and P. Gupta: Low-level laser therapy as an adjunct to conventional therapy in the treatment of diabetic foot ulcers. Lasers Med Sci, 2017. 32(2), 275-282.

27. Denadai, A.S., R.D. Aydos, I.S. Silva, L. Olmedo, B.M. de Senna Cardoso, B.A.K. da Silva, et al.: Acute effects of low-level laser therapy (660 $\mathrm{nm}$ ) on oxidative stress levels in diabetic rats with skin wounds. J Exp Therapeut Oncol, 2017. 11(2), 85-89.

28. Mirmiranpour, H., F.S. Nosrati, S.O. Sobhai, S.N. Takantape, and A. Amjadi: Effect of low-level laser irradiation on the function of glycated catalase. J Lasers Med Sci, 2018. 9(3), 212.

29. Lim, J., R.A. Sanders, A.C. Snyder, J.T. Eells, D.S. Henshel, and J.B. Watkins III: Effects of low-level light therapy on streptozotocin-induced diabetic kidney. J Photochem Photobiol B, 2010. 99(2), 105-110.

30. Longo, L., M. Postiglione, T. Buccioni, and D. Longo. The effects of Low Level LASER Therapy (LLLT) on blood glucose levels in patients with Diabetes Mellitus type I: a case report. in AIP Conference Proceedings. 2009. American Institute of Physics.
31. Gur, J., M. Mawuntu, and D. Martirosyan: FFC's Advancement of Functional Food Definition. Funct Food Health Dis, 2018. 8(7), 385-397.

32. Louis-Jean, S. and D. Martirosyan: Nutritionally attenuating the human gut microbiome to prevent and manage metabolic syndrome. J Agric Food Chem, 2019. 67(46), 12675-12684.

33. Silva, F.S., P.J. Oliveira, and M.F. Duarte: Oleanolic, ursolic, and betulinic acids as food supplements or pharmaceutical agents for type 2 diabetes: promise or illusion? J Agric Food Chem, 2016. 64(15), 2991-3008.

34. Chabosseau, P. and Rutter, G., A. Zinc and diabetes. Arch Biochem Biophys, 2016. 611, 79-85.

35. Sun, W., J. Yang, W. Wang, J. Hou, Y. Cheng, Y. Fu, et al.: The beneficial effects of $\mathrm{Zn}$ on Akt-mediated insulin and cell survival signaling pathways in diabetes. J Trace Elem Med Biol, 2018. 46, 117-127.

36. Abdel-Magied, N., A.A. Elkady, and S.M.A. Fattah: Effect of Low-Level Laser on Some Metals Related to Redox State and Histological Alterations in the Liver and Kidney of Irradiated Rats. Biol Trace Elem Res, 2020. 194(2), 410-422.

37. Guarino, G., G. Ragozzino, T. Della Corte, S. Fontana, and F. Strollo: Selenium supplementation in obese patients with subclinical hypothyroidism and type 2 diabetes. J Nutri Health Sci, 2018. 5(2), 202.

38. Atalay, H., Boyuk, B., Altay, M., Kiziler, A.R., and B. Aydemir: Serum Trace Elements in Type 2 Diabetes Mellitus. Acta Medica Mediterr, 2017, 33(75), 795-800.

39. Atalay, H., B. Boyuk, S. Guzel, M. Altay, A.R. Kiziler, and B. Aydemir: Serum trace elements in type 2 diabetes mellitus. Acta Medica, 2017. 33, 795. 